\title{
Genomic imprinting in mammals: its life cycle, molecular mechanisms and reprogramming
}

\author{
Yufeng $\mathrm{Li}^{1}$, Hiroyuki Sasaki ${ }^{1}$ \\ ${ }^{1}$ Division of Epigenomics, Department of Molecular Genetics, Medical Institute of Bioregulation, Kyushu University, 3-1-1 Mai- \\ dashi, Higashi-ku, Fukuoka 812-8582, Japan
}

Genomic imprinting, an epigenetic gene-marking phenomenon that occurs in the germline, leads to parental-origin-specific expression of a small subset of genes in mammals. Imprinting has a great impact on normal mammalian development, fetal growth, metabolism and adult behavior. The epigenetic imprints regarding the parental origin are established during male and female gametogenesis, passed to the zygote through fertilization, maintained throughout development and adult life, and erased in primordial germ cells before the new imprints are set. In this review, we focus on the recent discoveries on the mechanisms involved in the reprogramming and maintenance of the imprints. We also discuss the epigenetic changes that occur at imprinted loci in induced pluripotent stem cells.

Keywords: genomic imprinting; reprogramming; DNA methylation; DNA demethylation; histone modification

Cell Research (2011) 21:466-473. doi:10.1038/cr.2011.15; published online 1 February 2011

\section{Introduction}

In diploid organisms, the maternal and paternal alleles of most autosomal genes are expressed at similar levels, and thus contribute equally to the phenotype. However, in eutherian mammals (such as humans and mice) and marsupials, the parental alleles are not always functionally equivalent. This was first discovered in early $1980 \mathrm{~s}$ by embryological studies in mice: nuclear transfer experiments using pronuclear stage embryos showed that reconstituted embryos with two maternal genomes and no paternal complement and those with two paternal genomes and no maternal complement never survive beyond mid-gestation. This suggested that the parental genomes are functionally non-equivalent and marked or imprinted differently during male and female gametogenesis $[1,2]$. Almost at the same time, genetic experiments using chromosome translocations in mice showed that specific chromosomal segments, but not the entire genome, function differently depending on the parental origin [3]. Then, mouse $\operatorname{Ig} f 2 r$ was identified as the first imprinted gene in 1991: it was expressed only from the

Correspondence: Hiroyuki Sasaki

Fax: +81-92-642-6799

E-mail: hsasaki@bioreg.kyushu-u.ac.jp maternal allele [4]. To date, more than 100 imprinted genes have been identified in mice (http://www.mousebook.org/catalog.php?catalog=imprinting), and many of them are also imprinted in humans [5]. All imprinted genes show either maternal-specific or paternal-specific mono-allelic expression, and their proper expression is essential for normal development, fetal growth, nutrient metabolism and adult behavior. In humans, genetic and epigenetic disturbances in expression of the imprinted genes can cause well-known malformation disorders, such as Prader-Willi syndrome, Angelman syndrome, Beckwith-Wiedemann syndrome and Silver-Russell syndrome [5-7].

Most of the imprinted genes are found in clusters in the genome, corresponding to the specific chromosomal segments identified by the above genetic studies. Such imprinted clusters often span hundreds to thousands of kilobases. A given imprinted cluster can comprise both paternally and maternally expressed imprinted genes, some of which correspond to non-coding RNAs, and also non-imprinted genes [8-10]. The clusters also contain $\mathrm{CpG}$-rich regions that are DNA-methylated only on one of the two parental chromosomes (differentially methylated regions, DMRs). At some DMRs, differential DNA methylation is also observed between sperm and oocytes, and therefore gametic in origin. These DMRs are called germline or gametic DMRs. In some cases, there is evi- 
dence that the germline DMR functions as an imprinting control region, which controls the mono-allelic expression of the imprinted genes and the methylation status of the other DMRs within the cluster [11]. Most of the germline DMRs are methylated in the female germline and only four DMRs (H19, Dlk1-Gtl2, Rasgrfl and $Z d b f 2$ ) are known to be methylated in the male germline $[12,13]$. Importantly, mutations in the maintenance DNA methyltranferase DNMT1 disrupt the parental-originspecific expression patterns of the imprinted genes in mouse embryos [14]. In addition to DNA methylation, other epigenetic modifications and factors, such as histone modifications, insulator proteins (such as CTCF) and long non-coding RNAs, are also involved in imprinting.

The epigenetic modifications including DNA methylation at the germline DMRs undergo dynamic reprogramming during germ cell development but, on the other hand, they are maintained and faithfully propagated throughout embryonic development $[11,15,16]$. The whole process is complex and regulated tightly. In this study, we review the recent discoveries on the mechanisms involved in the establishment, maintenance and erasure of the epigenetic imprints. We also discuss the epigenetic changes observed at imprinted gene clusters in induced pluripotential stem (iPS) cells.

\section{Life cycle of the genomic imprints}

The life cycle of the genomic imprints in mammals is schematically shown in Figure 1. The cycle consists of three major steps: establishment, maintenance and erasure, all of which are important for this biological phenomenon. The establishment of the epigenetic imprints occurs in male and female germ cells. In the male germline, de novo DNA methylation of the four paternally methylated germline DMRs occurs progressively in mitotically arrested $(\mathrm{G} 1 / \mathrm{G} 0)$ prospermatogonia (or gonocytes) after embryonic day 14.5 (E14.5). Then, the paternal methylation imprints become fully established in prospermatogonia by the neonatal stage [17-21]. In the female germline, de novo DNA methylation initiates asynchronously at different germline DMRs during the oocyte growth phase $[22,23]$. Growing oocytes are at the diplotene/dictyate stage of meiotic prophase I, and the maternal methylation imprints become fully established by the fully grown oocyte stage $[22,23]$. The establishment of the maternal methylation imprints is correlated with the establishment of the functional imprints, which was shown by the developmental potential of nuclear transferred bi-maternal embryos [24].

The paternal and maternal epigenetic imprints es-

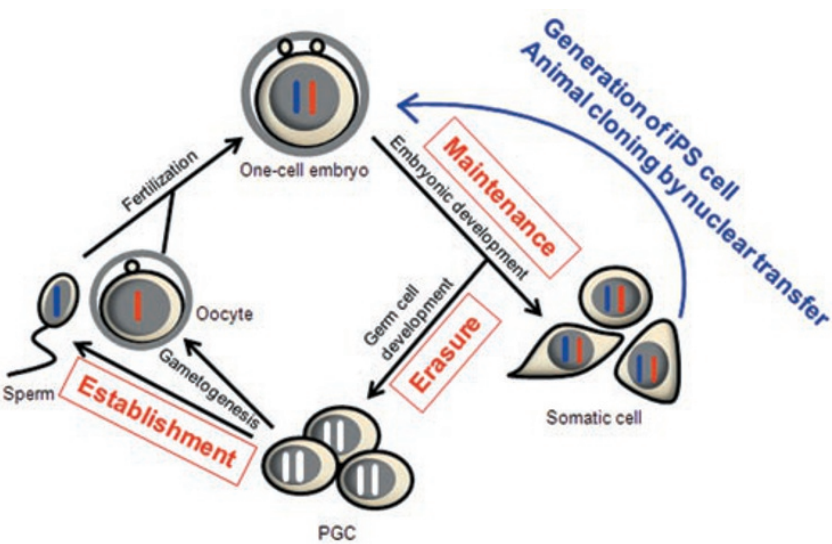

Figure 1 Life cycle of the genomic imprints. The paternal (blue) and maternal imprints (red) are established in the germ-line and maintained through fertilization and subsequent embryonic development. However, the imprints are erased in PGCs before the new imprints are set. The imprints need to be maintained during the extensive reprogramming that occurs in animal cloning and iPS cell generation (blue arrow).

tablished in the germline are transmitted to the zygote through fertilization and maintained faithfully throughout the development and adult life. Notably, the methylation imprints at the germline DMRs escape from the global epigenetic reprogramming that occurs in pre-implantation embryos $[11,16,25]$. The reprogramming at this stage includes the replacement of protamines by histones in the paternal genome, active demethylation of the paternal genome [26] and subsequent passive demethylation of both parental genomes [27, 28]. After implantation, the differential methylation at the germline DMRs has to survive another global epigenetic change, i.e., de novo DNA methylation. While many genes including the pluripotency genes and germ-cell-specific genes become highly methylated in early post-implantation embryos, the unmethylated allele of the DMR has to be protected from this strong wave of de novo DNA methylation. In fact, imprint maintenance is critical for the parentalorigin-specific mono-allelic expression of the imprinted genes throughout development.

The last step of the imprint life cycle is the erasure of the epigenetic imprints in primordial germ cells (PGCs): this ensures the sex-dependent imprint establishment in later stages of germ cell development described above. PGCs are specified from the epiblast cells of early postimplantation embryos. Then, PGCs proliferate actively, followed by migration to the genital ridge, the precursor of the gonads, between E7.25 and E10.5. In this period, the genome of the PGCs undergoes epigenetic reprogramming to restore pluripotency $[25,29,30]$, but they 


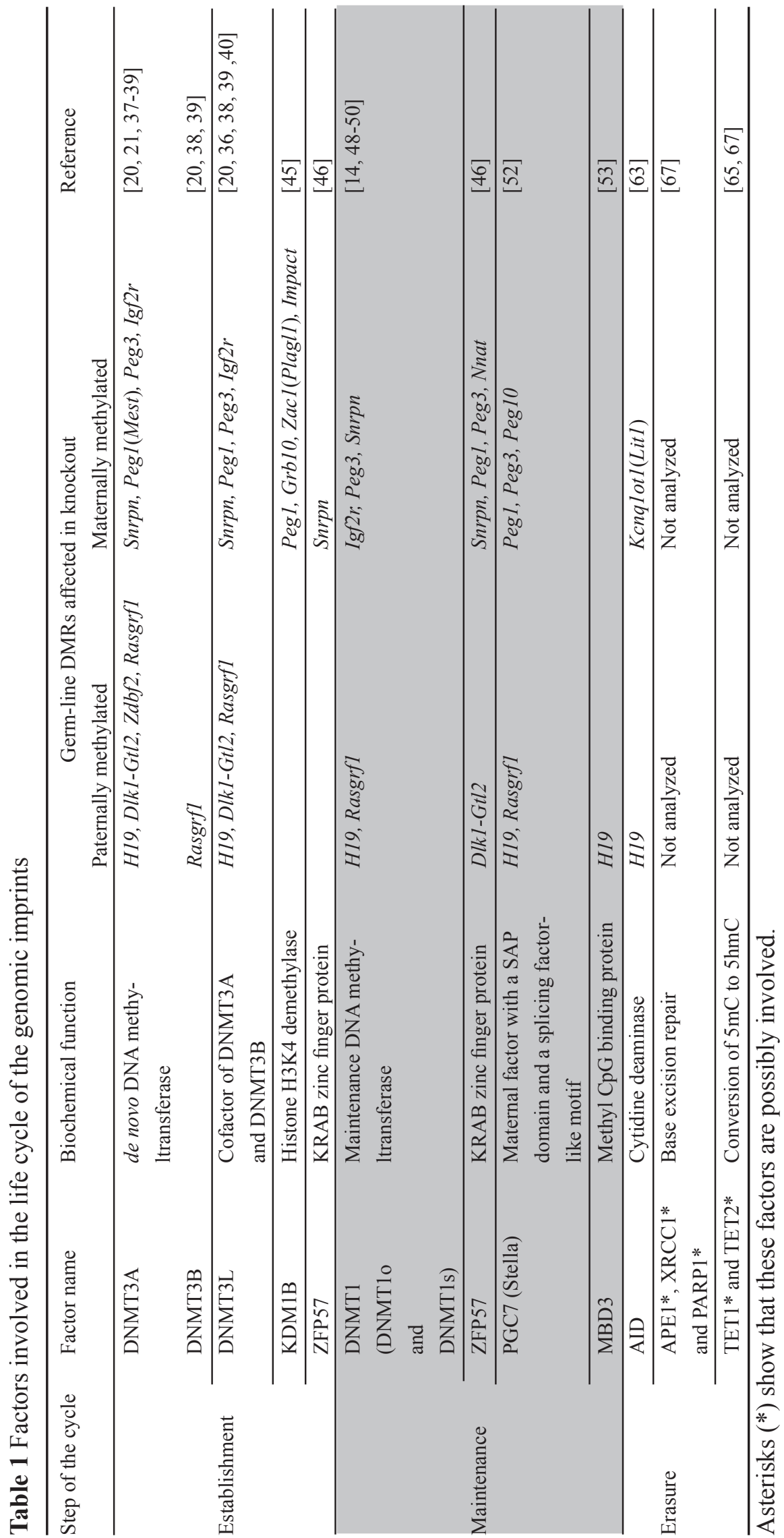


appear to retain the functional imprints at most DMRs [31]. Between E10.5 and E12.5, the parental-origin-specific DNA methylation is erased asynchronously at different germline DMRs, and the imprinted genes become biallelically expressed or silenced $[32,33]$. Consistent with this, the male and female embryonic germ cells derived at this stage have lost the parental-origin-specific DNA methylation at most DMRs [34].

Below we discuss the molecular mechanisms and factors involved in each step of the imprint life cycle. These factors are summarized in Table 1, together with their biochemical functions and target imprinted genes.

\section{Mechanism of imprint establishment in male and female germ cells}

Although circumstantial evidence showed that the gamete-specific differential DNA methylation at the germline DMRs is the functional imprints, direct evidence for this was lacking for a long time. The identification of the de novo DNA methyltransferase family genes dramatically changed this situation. Mammals have two active de novo DNA methyltransferases, namely, DNMT3A and DNMT3B [35], and a related protein, namely, DNMT3L $[36,37]$. DNMT3L has no methyltransferase activity, but is highly expressed in germ cells and can form a complex with DNMT3A and DNMT3B. When the genes coding for these proteins were respectively knocked out in the germline of mice, it was found that DNMT3A and DNMT3L are required for the establishment of the maternal imprints in growing oocytes [36-39]. In these studies, embryos derived from the mutant oocytes displayed loss of DNA methylation at the maternal alleles of the DMRs that are normally maternally methylated, and biallelic expression or silencing of the imprinted genes associated with these DMRs [36-39]. It was later confirmed that the mutant oocytes indeed lack DNA methylation at these germline DMRs [39]. It was also established that DNMT3B is dispensable for the establishment of the maternal imprints [39].

In the male germline, DNMT3A and DNMT3L again play a central role in de novo DNA methylation of the germline DMRs. In the Dnmt3a mutant prospermatogonia, all four paternally methylated germline DMRs showed reduced DNA methylation [20, 21, 38]. DNMT3L was also required for the de novo DNA methylation of all DMRs examined [20, 38, 40]. By contrast, in the Dn$m t 3 b$ mutants, only the Rasgrf1 DMR was affected [20, 38]. However, both Dnmt3a mutants and Dnmt3L mutants displayed meiotic arrest and azoospermia, and thus it was not possible to assess the effect of the loss of DNA methylation at the DMRs on parental-origin-specific mono-allelic expression of the imprinted genes in the embryo.

The discovery that the DNMT3A/DNMT3L complex establishes the methylation patterns at the DMRs in both male and female germlines raised a question of how this complex finds its sex-specific targets. Although the exact mechanism is still unknown, some interesting findings have been reported. First, based on the structural analysis of the DNMT3A/DNMT3L complex, it has been proposed that DNA regions with an 8-10-nucleotide $\mathrm{CpG}$ interval are the preferred substrate of the DNMT3A/ DNMT3L complex [41-43]. However, this sequence feature is found not only in the germline DMRs but also in many other $\mathrm{CpG}$ islands [43]. Second, unmethylated $\mathrm{H} 3 \mathrm{~K} 4$ has been proposed to serve as the chromatin signature for the recognition by DNMT3L $[41,44]$. Indeed, a lysine H3K4 demethylase KDM1B has been shown to be required for the establishment of the maternal imprints at some DMRs (Peg1 (also called Mest), Grb10, Zacl (also called Plagll) and Impact) [45]. However, this protein was dispensable for de novo DNA methylation of other DMRs examined (Kcnq1ot1 (also called Lit1), Igf $2 r$ and Snrpn). Third, a KRAB zinc-finger protein, ZFP57, has been shown to be required for the establishment of the DNA methylation imprint at the Snrpn DMR in oocytes [46]. However, this protein was dispensable for DNA methylation of other DMRs in oocytes and, furthermore, the functional imprint of the Snrpn DMR was preserved or restored after fertilization. Forth, a truncation of the Nesp transcripts at the Gnas locus in oocytes resulted in the loss of DNA methylation of the germline DMR, indicating that transcription through the DMR may be necessary to create or maintain an open chromatin environment that allows the DNMT3A/DNMT3L complex to gain access to its targets [47]. As the authors found such transcripts in other maternally methylated germline DMRs as well, they propose that this may be a common event for the establishment of the maternal methylation imprints in oocytes. Altogether, the mechanism underlying the recruitment of the DNMT3A/DNMT3L complex to specific targets seems complex, and the specificity may be determined by the combination of common factors and locus-specific factors.

\section{Factors involved in imprint maintenance}

Once established, the epigenetic imprints must be faithfully inherited to the zygote and maintained throughout embryonic development. The imprint maintenance is particularly important in pre-implantation embryos because it has to operate against the wave of genome-wide epigenetic reprogramming. First, the oocyte-specific 
isoform of the maintenance methyltransferase DNMT1, called DNMT1o, maintains the imprints at one single cell cycle in pre-implantation development [48]. Thus, the embryos derived from the oocytes lacking DNMT1o exhibited loss of DNA methylation at the germline DMRs and altered expression of the associated imprinted genes in about half of the cells [48]. More recently, it was reported that the zygotically expressed, somatic form of DNMT1, called DNMT1s, maintains the methylation imprints at the other cell cycles of pre-implantation development $[49,50]$. At present we do not know how the DNMT1 isoforms specifically find the DMRs among many other DNA regions, but a recent study suggested that a mammalian-specific region near the amino terminus of DNMT1 is probably involved in the discrimination [51].

Other than DNMT1, the following proteins may also have a role in the imprint maintenance in early embryos. First, ZFP57, an oocyte protein required for de novo DNA methylation of the Snrpn DMR, was shown to be present as a maternal protein in early embryos and essential for the maintenance of DNA methylation at several paternally and maternally methylated germline DMRs [46]. Second, PGC7 (also called Stella), another maternal protein, was shown to protect some germline DMRs from being reprogrammed in pre-implantation embryos [52], but how this multidomain protein achieve this is unknown. Third, a methyl-CpG-binding protein, MBD3, has a role in maintaining the paternal methylation imprint at the H19 DMR in pre-implantation embryos [53]. This appears to involve the recruitment of the Mi-2/NuRD repression complex to the highly CpG-methylated paternal allele of the H19 DMR. However, MBD3 depletion did not affect other imprinted genes examined, and therefore the involvement of this protein seems to be locus specific.

After implantation, the maintenance of the imprints requires DNMT1s in somatic lineages [14]. In addition to DNA methylation, however, the DMRs are also marked by differential histone modifications: the less CpG-methylated allele is marked by $\mathrm{H} 3 \mathrm{~K} 4 \mathrm{me}$ and histone acetylation, while the more $\mathrm{CpG}$-methylated allele is marked by $\mathrm{H} 3 \mathrm{~K} 9 \mathrm{me} 3, \mathrm{H} 4 \mathrm{~K} 20 \mathrm{me} 3$ and $\mathrm{H} 2 \mathrm{~A} / \mathrm{H} 4 \mathrm{R} 3 \mathrm{me} 2$ $[54,55]$. Interestingly, DNA methylation seems to be less important for the imprint maintenance in the trophoblast (placenta). This was first demonstrated at the Ascl2 (also called Mash2) locus: the maternal-specific expression of Ascl2 was maintained in the trophoblast lacking DNMT1 [56]. Later, it was shown that mutations in Dnmt1 do not cause loss of imprinting of the placenta-specific genes in an imprinted cluster on mouse chromosome 7 [57]. Further studies showed that the silent paternal alleles are marked by repressive histone modifications such as H3K9me2, mediated by G9a, and H3K27me3, mediated by the Polycomb repressive complex 2 (PRC2) $[57,58]$. Indeed, mice lacking G9a lose the mono-allelic expression patterns of the placenta-specific genes [59]. Also, in embryos lacking Eed, a component of the PRC2 complex, a subset of the paternally repressed genes was aberrantly activated in the trophoblast [60]. These observations highlight the importance of histone modifications in the imprint maintenance, but whether these marks are also present at imprinted regions in germ cells and/or gametes (especially in oocytes) is yet to be determined.

\section{Mechanism of imprint erasure in PGCs}

The erasure of the imprints in PGCs is most likely reflected by DNA demethylation. It can occur in an active or a passive way, but the rapid DNA demethylation in PGCs suggests that it might be an active process [33]. Although there are various possible mechanisms for active DNA demethylation [61], recent studies have provided clues to the demethylating mechanism in PGCs. The activation-induced cytidine deaminase (AID), which is expressed in tissues where demethylation occurs, was shown to be capable of deaminating 5-methylcytosine $(5 \mathrm{mC})$ to thymidine $(\mathrm{T})$ in DNA [62]. The resulting T-G mismatch might trigger a DNA repair pathway that results in the loss of $5 \mathrm{mC}$. Both genome-wide and locusspecific analyses of AID-deficient PGCs demonstrated that AID contributes to global demethylation, and also demethylation at some imprinted DMRs (H19 and $\mathrm{Kcn}$ q1ot1) in both male and female PGCs [63]. Nevertheless, considerable DNA demethylation still occurs in PGCs deficient for AID, indicating the presence of other demethylation mechanisms.

The 10-11 translocation family proteins (TET1, TET2 and TET3) catalyze the conversion of $5 \mathrm{mC}$ to 5 -hydroxymethylcytosine $(5 \mathrm{hmC})$ in vitro and in vivo [64, $65]$. The $5 \mathrm{hmC}$ may facilitate passive DNA demethylation by excluding proteins involved in maintenance methylation such as DNMT1 [66] or may represent an intermediate in an active demethylation pathway [61]. Since TET1 and TET2 are significantly expressed in PGCs at E11.5 and E12.5 [67], when the imprinted DMRs undergo demethylation, it is possible that the TET family proteins play a role in the erasure of the imprints. For example, if $5 \mathrm{hmC}$ is recognized by a glycosylase, then the base excision repair (BER) pathway may restore the unmethylated state, as DNA demethylation in the PGCs is accompanied by the appearance of single-stranded DNA breaks and the activation of the BER components [67]. Further studies are needed to fully understand the precise 
mechanism of DNA demethylation and imprint erasure in PGCs.

\section{Genomic imprinting and cell reprogramming tech- nology}

Recent advancement in the cell reprogramming technology showed that somatic cell nuclei of differentiated states can be reprogrammed to a pluripotent state either by nuclear transfer or by using defined factors $[68,69]$. In such a reprogramming process, pluripotency genes, developmental genes and tissue-specific genes are reprogrammed, but the parental-origin-specific epigenetic imprints, which ensure the mono-allelic expression of the imprinted genes, need to be maintained (Figure 1). It is unknown how the imprints at the DMRs escape from the global reprogramming, but errors in the imprint maintenance could be related to a reduced pluripotency, which is one of the major obstacles in iPS cell research.

Recently, it was reported that the expression state of the imprinted Dlk1-Dio3 cluster on mouse chromosome 12 is often altered in iPS cells and can be used as a marker to evaluate pluripotency [70]. In the affected iPS cell clones, a few imprinted genes, such as $G t l 2$, within the Dlk1-Dio3 cluster were abnormally silenced. Furthermore, these iPS cell clones contributed poorly to chimaeras and failed to support the development of entirely iPS cell-derived mice, whereas embryos derived from iPS cell clones with normal expression of these genes developed well [70]. The abnormalities at the Dlk1-Dio3 cluster were not seen in embryonic stem cells. In the iPS cell clones with silenced Gtl2, DNA hyper-methylation and histone hypo-acethylation were detected at the DMRs within the cluster. Since these DMRs are normally methylated only on the paternal chromosome [71], the observed abnormalities are viewed as a "paternalization" of the maternal chromosome. In other words, the unmethylated state of the maternally derived DMRs was not maintained. At present, the precise cause of this aberrant silencing is unknown, but the reprogramming procedure itself seems to induce these epigenetic changes [70]. Since the aberrant silencing of the Dlk1-Dio3 cluster is not frequent in cloned mice produced by nuclear transfer, the oocyte cytoplasm may contain a factor that protects the DMRs of this cluster from de novo DNA methylation. Clone-specific variations in the stability of mono-allelic expression of the imprinted genes were also reported in human iPS cells, but in this case various genes were affected (for example, $H 19$ and $K C N Q 1 O T 1$ ) [72].

\section{Outlook}

Genomic imprinting is an excellent model system to study nuclear reprogramming in mammals because the epigenetic imprints regarding the parental origin are fully reprogrammed in each generation. In the last 10 years or so, many factors involved in each step of the imprinting cycle were identified, and we started to learn how this interesting phenomenon occurs. Nevertheless, there remain many unanswered questions, e.g., how the regulatory factors identify specific targets for imprint establishment in the germline, how the imprints escape from genomewide reprogramming in pre-implantation embryos and how the imprints are erased in PGCs. Furthermore, an interesting link between the epigenetic aberrations in imprinted gene clusters and reduced developmental potential has been discovered in mouse iPS cells. Thus the studies on the mechanisms underlying each step of the life cycle of the genomic imprints should contribute to the improvement of the reprogramming technology for animal cloning and iPS cell generation.

\section{Acknowledgments}

This work was supported in part by Grants-in-Aid for Scientific Research on Priority Area from the Ministry of Education, Culture, Sports, Science and Technology of Japan.

\section{References}

1 McGrath J, Solter D. Completion of mouse embryogenesis requires both the maternal and paternal genomes. Cell 1984; 37:179-183.

2 Surani MA, Barton SC, Norris ML. Development of reconstituted mouse eggs suggests imprinting of the genome during gametogenesis. Nature 1984; 308:548-550.

3 Cattanach BM, Kirk M. Differential activity of maternally and paternally derived chromosome regions in mice. Nature 1985; 315:496-498.

4 Barlow DP, Stoger R, Herrmann BG, Saito K, Schweifer N. The mouse insulin-like growth factor type-2 receptor is imprinted and closely linked to the Tme locus. Nature 1991; 349:84-87.

5 Morison IM, Ramsay JP, Spencer HG. A census of mammalian imprinting. Trends Genet 2005; 21:457-465.

6 Walter J, Paulsen M. Imprinting and disease. Semin Cell Dev Biol 2003; 14:101-110.

7 Butler MG. Genomic imprinting disorders in humans: a minireview. J Assist Reprod Genet 2009; 26:477-486.

8 Thorvaldsen JL, Bartolomei MS. SnapShot: imprinted gene clusters. Cell 2007; 130:958.

9 Royo H, Cavaille J. Non-coding RNAs in imprinted gene clusters. Biol Cell 2008; 100:149-166.

10 Peters J, Robson JE. Imprinted noncoding RNAs. Mamm Genome 2008; 19:493-502.

11 Reik W, Walter J. Genomic imprinting: parental influence on the genome. Nat Rev Genet 2001; 2:21-32.

12 Reik W, Walter J. Evolution of imprinting mechanisms: the battle of the sexes begins in the zygote. Nat Genet 2001; 27:255-256. 
13 Arnaud P. Genomic imprinting in germ cells: imprints are under control. Reproduction 2010; 140:411-423.

14 Li E, Beard C, Jaenisch R. Role for DNA methylation in genomic imprinting. Nature 1993; 366:362-365.

15 Ferguson-Smith AC, Surani MA. Imprinting and the epigenetic asymmetry between parental genomes. Science 2001; 293:1086-1089.

16 Morgan HD, Santos F, Green K, Dean W, Reik W. Epigenetic reprogramming in mammals. Hum Mol Genet 2005; 14 Spec No 1:R47-58.

17 Davis TL, Yang GJ, McCarrey JR, Bartolomei MS. The H19 methylation imprint is erased and re-established differentially on the parental alleles during male germ cell development. Hum Mol Genet 2000; 9:2885-2894.

18 Ueda T, Abe K, Miura A, et al. The paternal methylation imprint of the mouse H19 locus is acquired in the gonocyte stage during foetal testis development. Genes Cells 2000; 5:649-659.

19 Li JY, Lees-Murdock DJ, Xu GL, Walsh CP. Timing of establishment of paternal methylation imprints in the mouse. Genomics 2004; 84:952-960.

20 Kato Y, Kaneda M, Hata K, et al. Role of the Dnmt3 family in de novo methylation of imprinted and repetitive sequences during male germ cell development in the mouse. Hum Mol Genet 2007; 16:2272-2280.

21 Hiura H, Sugawara A, Ogawa $\mathrm{H}$, et al. A tripartite paternally methylated region within the Gpr1-Zdbf2 imprinted domain on mouse chromosome 1 identified by meDIP-on-chip. Nucleic Acids Res 2010; 38:4929-4945.

22 Lucifero D, Mann MR, Bartolomei MS, Trasler JM. Genespecific timing and epigenetic memory in oocyte imprinting. Hum Mol Genet 2004; 13:839-849.

23 Hiura H, Obata Y, Komiyama J, Shirai M, Kono T. Oocyte growth-dependent progression of maternal imprinting in mice. Genes Cells 2006; 11:353-361.

24 Kono T, Obata Y, Yoshimzu T, Nakahara T, Carroll J. Epigenetic modifications during oocyte growth correlates with extended parthenogenetic development in the mouse. Nat Genet 1996; 13:91-94.

25 Sasaki H, Matsui Y. Epigenetic events in mammalian germcell development: reprogramming and beyond. Nat Rev Genet 2008; 9:129-140.

26 Oswald J, Engemann S, Lane N, et al. Active demethylation of the paternal genome in the mouse zygote. Curr Biol 2000; 10:475-478.

27 Rougier N, Bourc'his D, Gomes DM, et al. Chromosome methylation patterns during mammalian preimplantation development. Genes Dev 1998; 12:2108-2113.

28 Santos F, Hendrich B, Reik W, Dean W. Dynamic reprogramming of DNA methylation in the early mouse embryo. Dev Biol 2002; 241:172-182.

29 Seki Y, Yamaji M, Yabuta Y, et al. Cellular dynamics associated with the genome-wide epigenetic reprogramming in migrating primordial germ cells in mice. Development 2007; 134:2627-2638.

30 Surani MA, Hayashi K, Hajkova P. Genetic and epigenetic regulators of pluripotency. Cell 2007; 128:747-762.

31 Szabo PE, Hubner K, Scholer H, Mann JR. Allele-specific expression of imprinted genes in mouse migratory primordial germ cells. Mech Dev 2002; 115:157-160.
32 Lee J, Inoue K, Ono R, et al. Erasing genomic imprinting memory in mouse clone embryos produced from day 11.5 primordial germ cells. Development 2002; 129:1807-1817.

33 Hajkova P, Erhardt S, Lane N, et al. Epigenetic reprogramming in mouse primordial germ cells. Mech Dev 2002; 117:1523.

34 Tada T, Tada M, Hilton K, et al. Epigenotype switching of imprintable loci in embryonic germ cells. Dev Genes Evol 1998; 207:551-561.

35 Okano M, Bell DW, Haber DA, Li E. DNA methyltransferases Dnmt3a and Dnmt3b are essential for de novo methylation and mammalian development. Cell 1999; 99:247-257.

36 Bourc'his D, Xu GL, Lin CS, Bollman B, Bestor TH. Dnmt3L and the establishment of maternal genomic imprints. Science 2001; 294:2536-2539.

37 Hata K, Okano M, Lei H, Li E. Dnmt3L cooperates with the Dnmt3 family of de novo DNA methyltransferases to establish maternal imprints in mice. Development 2002; 129:1983-1993.

38 Kaneda M, Okano M, Hata K, et al. Essential role for de novo DNA methyltransferase Dnmt3a in paternal and maternal imprinting. Nature 2004; 429:900-903.

39 Kaneda M, Hirasawa R, Chiba H, Okano M, Li E, Sasaki H. Genetic evidence for Dnmt3a-dependent imprinting during oocyte growth obtained by conditional knockout with Zp3-Cre and complete exclusion of Dnmt3b by chimera formation. Genes Cells 2010 Feb 1. doi: 10.1111/j.1365-2443.2009.01374.x

40 Bourc'his D, Bestor TH. Meiotic catastrophe and retrotransposon reactivation in male germ cells lacking Dnmt3L. Nature 2004; 431:96-99.

41 Jia D, Jurkowska RZ, Zhang X, Jeltsch A, Cheng X. Structure of Dnmt3a bound to Dnmt3L suggests a model for de novo DNA methylation. Nature 2007; 449:248-251.

42 Jurkowska RZ, Anspach N, Urbanke C, et al. Formation of nucleoprotein filaments by mammalian DNA methyltransferase Dnmt3a in complex with regulator Dnmt3L. Nucleic Acids Res 2008; 36:6656-6663.

43 Glass JL, Fazzari MJ, Ferguson-Smith AC, Greally JM. CG dinucleotide periodicities recognized by the Dnmt3a-Dnmt3L complex are distinctive at retroelements and imprinted domains. Mamm Genome 2009; 20:633-643.

44 Ooi SK, Qiu C, Bernstein E, et al. DNMT3L connects unmethylated lysine 4 of histone $\mathrm{H} 3$ to de novo methylation of DNA. Nature 2007; 448:714-717.

45 Ciccone DN, Su H, Hevi S, et al. KDM1B is a histone H3K4 demethylase required to establish maternal genomic imprints. Nature 2009; 461:415-418.

46 Li X, Ito M, Zhou F, et al. A maternal-zygotic effect gene, Zfp57, maintains both maternal and paternal imprints. Dev Cell 2008; 15:547-557.

47 Chotalia M, Smallwood SA, Ruf N, et al. Transcription is required for establishment of germline methylation marks at imprinted genes. Genes Dev 2009; 23:105-117.

48 Howell CY, Bestor TH, Ding F, et al. Genomic imprinting disrupted by a maternal effect mutation in the Dnmt1 gene. Cell 2001; 104:829-838.

49 Kurihara Y, Kawamura Y, Uchijima Y, et al. Maintenance of genomic methylation patterns during preimplantation development requires the somatic form of DNA methyltransferase 1. Dev Biol 2008; 313:335-346. 
50 Hirasawa R, Chiba H, Kaneda M, et al. Maternal and zygotic Dnmtl are necessary and sufficient for the maintenance of DNA methylation imprints during preimplantation development. Genes Dev 2008; 22:1607-1616.

51 Borowczyk E, Mohan KN, D'Aiuto L, Cirio MC, Chaillet JR. Identification of a region of the DNMT1 methyltransferase that regulates the maintenance of genomic imprints. Proc Natl Acad Sci USA 2009; 106:20806-20811.

52 Nakamura T, Arai Y, Umehara H, et al. PGC7/Stella protects against DNA demethylation in early embryogenesis. Nat Cell Biol 2007; 9:64-71.

53 Reese KJ, Lin S, Verona RI, Schultz RM, Bartolomei MS. Maintenance of paternal methylation and repression of the imprinted H19 gene requires MBD3. PLoS Genet 2007; 3:e137.

54 Fournier C, Goto Y, Ballestar E, et al. Allele-specific histone lysine methylation marks regulatory regions at imprinted mouse genes. EMBO J 2002; 21:6560-6570.

55 Henckel A, Nakabayashi K, Sanz LA, Feil R, Hata K, Arnaud P. Histone methylation is mechanistically linked to DNA methylation at imprinting control regions in mammals. Hum Mol Genet 2009; 18:3375-3383.

56 Tanaka M, Puchyr M, Gertsenstein M, et al. Parental originspecific expression of Mash2 is established at the time of implantation with its imprinting mechanism highly resistant to genome-wide demethylation. Mech Dev 1999; 87:129-142.

57 Lewis A, Mitsuya K, Umlauf D, et al. Imprinting on distal chromosome 7 in the placenta involves repressive histone methylation independent of DNA methylation. Nat Genet 2004; 36:1291-1295.

58 Umlauf D, Goto Y, Cao R, et al. Imprinting along the Kcnq1 domain on mouse chromosome 7 involves repressive histone methylation and recruitment of Polycomb group complexes. Nat Genet 2004; 36:1296-1300.

59 Wagschal A, Sutherland HG, Woodfine K, et al. G9a histone methyltransferase contributes to imprinting in the mouse placenta. Mol Cell Biol 2008; 28:1104-1113.

60 Mager J, Montgomery ND, de Villena FP, Magnuson T. Genome imprinting regulated by the mouse Polycomb group protein Eed. Nat Genet 2003; 33:502-507.

$61 \mathrm{Wu}$ SC, Zhang Y. Active DNA demethylation: many roads lead to Rome. Nat Rev Mol Cell Biol 2010; 11:607-620.

62 Morgan HD, Dean W, Coker HA, Reik W, Petersen-Mahrt SK. Activation-induced cytidine deaminase deaminates 5-methylcytosine in DNA and is expressed in pluripotent tissues: implications for epigenetic reprogramming. J Biol Chem 2004; 279:52353-52360.

63 Popp C, Dean W, Feng S, et al. Genome-wide erasure of DNA methylation in mouse primordial germ cells is affected by AID deficiency. Nature 2010; 463:1101-1105.

64 Tahiliani M, Koh KP, Shen Y, et al. Conversion of 5-methylcytosine to 5-hydroxymethylcytosine in mammalian DNA by MLL partner TET1. Science 2009; 324:930-935.

65 Ito S, D’Alessio AC, Taranova OV, Hong K, Sowers LC, Zhang Y. Role of Tet proteins in $5 \mathrm{mC}$ to $5 \mathrm{hmC}$ conversion, ES-cell self-renewal and inner cell mass specification. Nature 2010; 466:1129-1133.

66 Valinluck V, Sowers LC. Endogenous cytosine damage products alter the site selectivity of human DNA maintenance methyltransferase DNMT1. Cancer Res 2007; 67:946-950.

67 Hajkova P, Jeffries SJ, Lee C, Miller N, Jackson SP, Surani MA. Genome-wide reprogramming in the mouse germ line entails the base excision repair pathway. Science 2010; 329:7882.

68 Campbell KH, McWhir J, Ritchie WA, Wilmut I. Sheep cloned by nuclear transfer from a cultured cell line. Nature 1996; 380:64-66.

69 Takahashi K, Yamanaka S. Induction of pluripotent stem cells from mouse embryonic and adult fibroblast cultures by defined factors. Cell 2006; 126:663-676.

70 Stadtfeld M, Apostolou E, Akutsu H, et al. Aberrant silencing of imprinted genes on chromosome $12 \mathrm{qF} 1$ in mouse induced pluripotent stem cells. Nature 2010; 465:175-181.

71 Takada S, Paulsen M, Tevendale M, et al. Epigenetic analysis of the Dlk1-Gt12 imprinted domain on mouse chromosome 12: implications for imprinting control from comparison with Igf2-H19. Hum Mol Genet 2002; 11:77-86.

72 Pick M, Stelzer Y, Bar-Nur O, Mayshar Y, Eden A, Benvenisty N. Clone- and gene-specific aberrations of parental imprinting in human induced pluripotent stem cells. Stem Cells 2009; 27:2686-2690. 